\title{
Micro 2D-TLC of Selected Plant Extracts in Screening of Their Composition and Antioxidative Properties
}

\author{
Mirosław A. Hawrył • Monika Waksmundzka-Hajnos
}

Received: 30 January 2013/Revised: 23 April 2013/ Accepted: 25 May 2013/Published online: 29 June 2013

(C) The Author(s) 2013. This article is published with open access at Springerlink.com

\begin{abstract}
Micro two-dimensional separations were performed on polar bonded stationary phases of the type cyanopropyl-silica using non-aqueous eluents (polar modifier dissolved in $n$-heptane) as the first direction eluents and aqueous eluents (organic modifier- $\mathrm{MeOH}$ dissolved in water) as the second direction eluents. The chromatographic process was performed in micro scale using $5 \times 5 \mathrm{~cm}$ plates, small volumes of eluents and $10 \mu \mathrm{L}$ of plant extracts to obtain satisfying separation. Plates developed in horizontal chambers were dried and observed in UV light (254 $\mathrm{nm}$ and $366 \mathrm{~m})$ photographed by digital camera and derivatized by DPPH to detect antioxidants (free radical scavengers) or derivatized by Naturstoff reagent to detect phenolic compounds (characteristic luminescence of some phenolic compounds). The above experiments give the possibility to construct fingerprints for investigated Polygonum hydropiper, Betula verrucosa and Pulmonaria officinalis extracts. It can be used in quality control of the plant material and its antioxidative activity. Novelty of the paper is the micro-scale of the separation by two-dimensional thin layer chromatography mode. For the first time two-dimensional separation of plant extracts on $5 \times 5 \mathrm{~cm}$ plates in two directions is performed.
\end{abstract}

Published in the topical collection Miniaturized and New Featured Planar Chromatography and Related Techniques with guest editor Paweł K. Zarzycki.

M. A. Hawrył · M. Waksmundzka-Hajnos ( $\square)$

Department of Inorganic Chemistry, Faculty of Pharmacy,

Medical University of Lublin, Chodźki 4A,

20-093 Lublin, Poland

e-mail: monika.hajnos@am.lublin.pl
Keywords Micro thin layer chromatography - Twodimensional thin layer chromatography (2D-TLC) . Phenolic compounds · Plant extracts · Antioxidants

\section{Introduction}

The increasing demand of the development of analytical environmental friendly methods contributes to the miniaturization of existing and well-functioning analysis methods. The main advantage of micro methods (HPLC and TLC) is a low consumption of solvents and the fact that the separations are performed on small plates $(7 \times 7 \mathrm{~cm}$ or less). The separation process is realized on the way 5-7 cm, where theoretical plate height is minimal $[1,2]$. In this case, the time of analysis is shortened in comparison with classic TLC methods, where the separation is performed on $10-20 \mathrm{~cm}$ plates. There are some papers in which the great efficacy of micro-TLC was proved in the analysis of steroids, plant extracts, dyes from Spirulina maxima and some fullerenes [3-6].

The instrumentation for micro-TLC is as simple as in HPTLC and TLC. Classic horizontal chambers can be used. Zarzycki et al. [5] constructed the developing chamber for micro-TLC in which the volume of eluent used is $300-1,000 \mu \mathrm{L}$ depending on its viscosity.

Polygonum hydropiper $L$. also known as smartweed has a long history of herbal use, both in Eastern and Western herbalism. It is not very often used, and it is seen more as a domestic remedy being valued especially for its astringent properties, which makes it useful in treating bleeding, skin problems, diarrhea, etc. The leaves have anti-inflammatory, astringent, carminative, diaphoretic, diuretic, emmenagogue, stimulant, stomachic and styptic properties. They contain rutin, which helps strengthen fragile capillaries and 
thus helps prevent bleeding. The seeds are carminative, diuretic and stimulant. The whole plant, either on its own or mixed with other herbs, is decocted and used in the treatment of a wide range of ailments including diarrhea, dyspepsia, itching skin, excessive menstrual bleeding and hemorrhoids. A poultice of the plant is used in treating swollen and inflamed areas. In Chinese tests, the plant was ranked 20th in a survey of 250 potential antifertility drugs. A homeopathic remedy is made from the leaves. It is used in the treatment of piles, menstrual pains and other menstrual complaints $[7,8]$.

The leaves of the herb Betula verrucosa Ehrh. (also known as birch) should be dried in the shade at a temperature below $40{ }^{\circ} \mathrm{C}$. Also used for medicinal purposes are flowers, bark, young branches (falls) and tree sap. Among the medicinal compounds that the tree has, there are the flavonoids that give this material diuretic property. Birch is used in cases of urinary tract infections, such as cystitis, calculus, pyelonephritis, among others. In addition, the leaves have in essential oils such compounds as betulinol, which gives febrifuge actions. Birch is also a good antiseptic and healing remedy. The bark of B. verrucosa have tannins, which act as astringents. The sap acts as a diuretic and antirheumatic and is used in cases of gout and rheumatism [7].

Pulmonaria officinalis L. (also known as lungwort) has a high mucilage content and this makes it useful in the treatment of chest conditions, being of particular benefit in cases of chronic bronchitis. It combines well with other herbs such as coltsfoot (Tussilago farfara) in the treatment of chronic coughs including whooping cough and can also be taken to treat asthma. The leaves and flowering shoots are astringent, demulcent, diaphoretic, diuretic, emollient, mildly expectorant and resolvent. They are often used for their healing effect in pulmonary complaints and their mucilaginous nature makes them beneficial in treating sore throats. The leaves can also be used externally to stop bleeding. They are harvested in the spring and dried for later use. A fluid made from the plant is an effective eyewash for tired eyes. A homeopathic remedy is made from the plant. It is used in the treatment of bronchitis, coughs and diarrhea [7].

The main aim of this work is the examination of the possibility of using micro-TLC in two-dimensional separations of some medicinal plant extracts ( $P$. hydropiper, $B$. verrucosa and $P$. officinalis) to obtain the information about their composition and antioxidative activity. The antioxidative activity can be measured using 1,1-diphenyl2-picrylhydrazyl (DPPH) as spraying reagent for postchromatography derivatization [9]. The success of this investigations can give possibilities to use $5 \times 5 \mathrm{~cm}$ plates in two-dimensional thin layer chromatography (2D-TLC) experiments for screening of plant extracts for the presence of antioxidants in an easy and inexpensive way.

\section{Experimental}

The plant materials were obtained from the following producers: Herba Pulmonariae from Flos (Mokrsko, Poland), Herba Polygoni hydropiperis from Herbapol (Kraków, Poland) and Folium Betulae from Herbapol (Lublin, Poland).

Five grams of each plant material was weighted out. Each material was closed in the paper case and extracted in Soxhlet apparatus on water bath for $10 \mathrm{~h}$ using chloroform to isolate non polar constituents (chlorophylls). Dried plant materials (in the paper case) after the first extraction were once more extracted by methanol by portion of $300 \mathrm{~mL}$ during $10 \mathrm{~h}$. The methanolic extract was evaporated to dryness under reduced pressure in the vacuum evaporator. The dry residue was dissolved in methanol solvent and filled with the same solvent in $25 \mathrm{~mL}$ flasks. The prepared extracts were filtered using SOR25 0.45 filter and stored in refrigerator.

HPTLC CN F254s $10 \times 10 \mathrm{~cm}$ (Merck, Darmstadt, Germany) cut to the $5 \times 5 \mathrm{~cm}$ squares were used in all experiments. Solvents: propan-2-ol, tetrahydrofuran, $n$ heptane and methanol pro analysis grade were purchased from Polish Reagents (POCh, Gliwice, Poland). Distilled water was mixed with methanol to obtain aqueous phases and $n$-heptane was mixed with propan-2-ol, tetrahydrofuran or ethyl methyl ketone to obtain non-aqueous eluents for 2D-HPTLC.

All test substances listed in Table 1 were acquired from various manufacturers (Sigma, Aldrich, Fluka, Roth). 2,2'Diphenyl-1-picrylhydrazyl (DPPH) was from Aldrich and

Table 1 Substances investigated

\begin{tabular}{lll}
\hline & Substance & Manufacturer \\
\hline 1 & Naringin & Sigma \\
2 & Hesperidin & Aldrich \\
3 & Naringenin & Aldrich \\
4 & Hesperetin & Aldrich \\
5 & Flavone & Sigma \\
6 & Apigenin 7-glucoside & Roth \\
7 & Quercetin & Sigma \\
8 & Rutin & Sigma \\
9 & Chlorogenic acid & Aldrich \\
10 & Resveratrol & Roth \\
11 & Myricetin & Aldrich \\
12 & Hyperoside & Aldrich \\
13 & Acacetin & Aldrich \\
14 & Ferulic acid & Fluka \\
15 & Gallic acid & Fluka \\
16 & Caffeic acid & Fluka \\
17 & $p$-Coumaric acid & Fluka \\
\hline
\end{tabular}




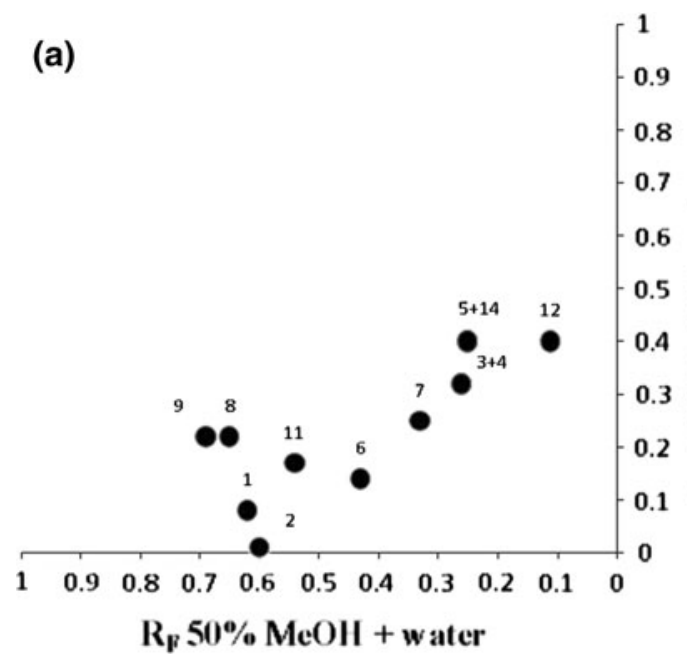

Fig. 1 a Simulated map of the separation of standards as a plot of $\mathrm{R}_{\mathrm{F}}$ vs. $\mathrm{R}_{\mathrm{F}}$ for the systems: $\mathrm{I}-40 \% \mathrm{iPrOH}+n-\mathrm{Hp} ; \mathrm{II}-50 \% \mathrm{MeOH}+$ water. Numbers as in Table $1 \mathbf{b}$ Simulated map of the separation of (b)

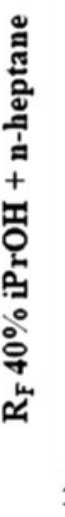

standards as a plot of $\mathrm{R}_{\mathrm{F}}$ vs. $\mathrm{R}_{\mathrm{F}}$ for the systems: $\mathrm{I}-50 \% \mathrm{THF}+n$ $\mathrm{Hp} ; \mathrm{II}-40 \% \mathrm{MeOH}+$ water. Numbers as in Table 1
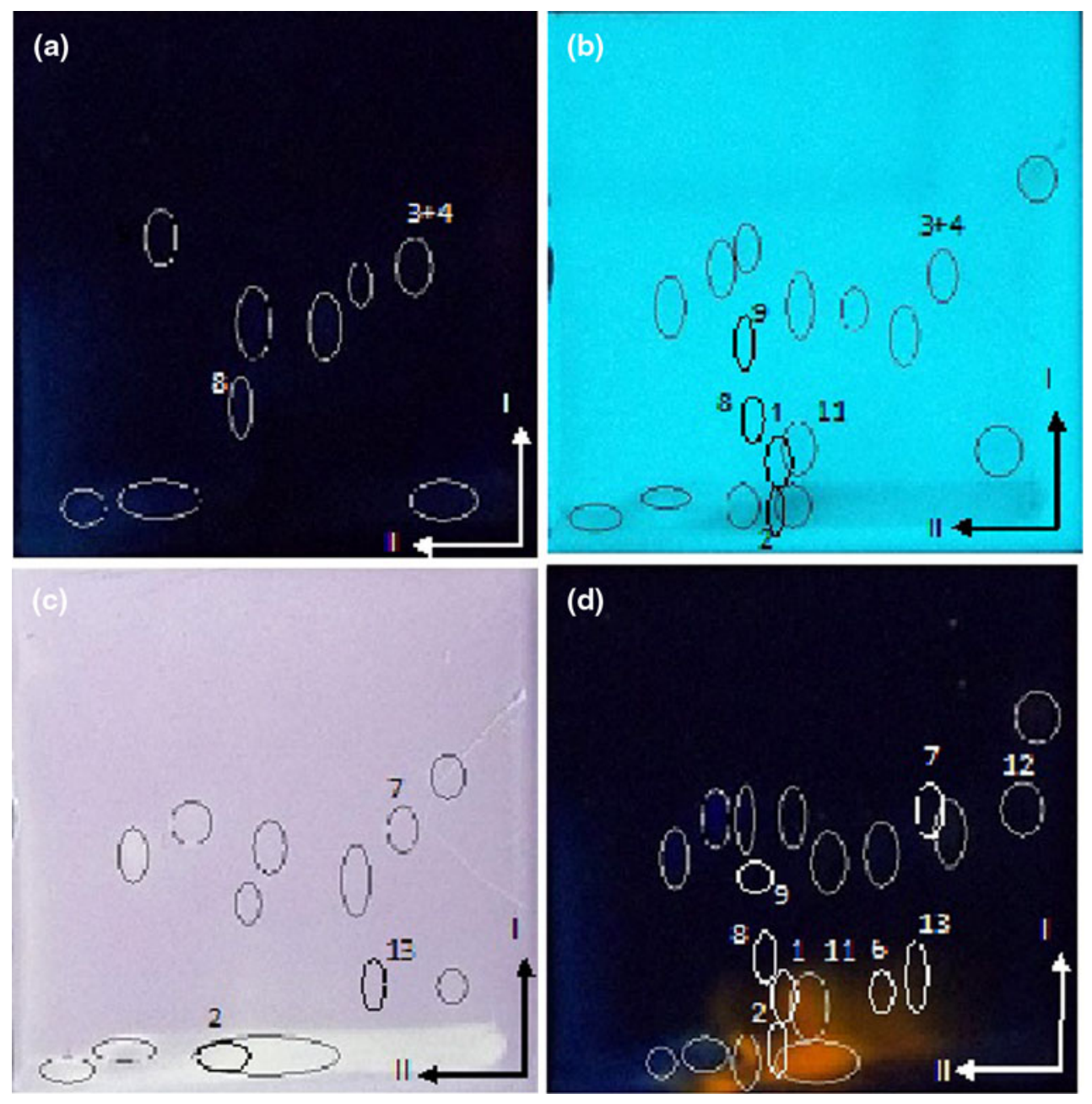

Fig. 2 Photographs of chromatographic cyanopropyl plates developed by 2D-TLC mode (I-50\% THF $+n$-Hp; II- $40 \% \mathrm{MeOH}+$ water) with separated extract of Polygonum hydropiper. a at

$\lambda=366 \mathrm{~nm} ; \mathbf{b}$ at $\lambda=254 \mathrm{~nm} \mathbf{c}$ after derivatization with DPPH in daylight $\mathbf{d}$ after derivatization with Naturstoff reagent at $\lambda=366 \mathrm{~nm}$. Numbers as in Table 1 
Fig. 3 Photographs of chromatographic cyanopropyl plates developed by $2 \mathrm{D}$-TLC mode $(\mathrm{I}-40 \%$ iPrOH $+n-\mathrm{Hp}$; $\mathrm{II}-50 \% \mathrm{MeOH}+$ water) with separated extract of Betula verrucosa. a at $\lambda=366 \mathrm{~nm}$; b at $\lambda=254 \mathrm{~nm}$ c after derivatization with DPPH in daylight $\mathbf{d}$ after derivatization with Naturstoff reagent at $\lambda=366 \mathrm{~nm}$. Numbers as in Table 1
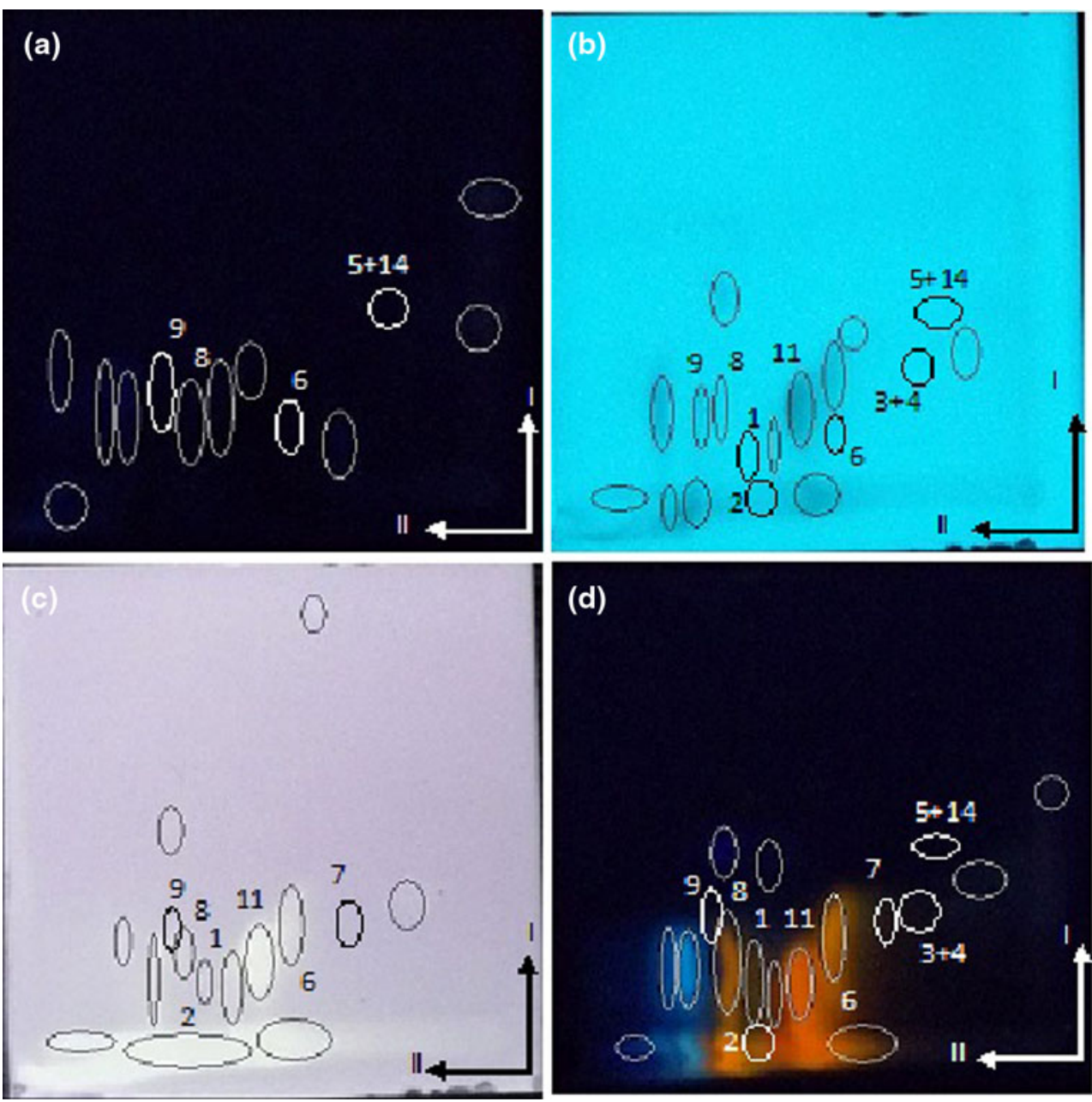

used as $0.2 \%$ solution in methanol. 2-(Diphenylboryoxy)ethylamine and PEG4000-Naturstoff reagent was produced by Merck (Darmstadt, Germany) and used as $5 \%$ methanolic solution of PEG and $1 \%$ methanolic solution of 2-(diphenylboryoxy)-ethylamine.

The $0.1 \%(w / v)$ solution of the mixture of test substances $(10 \mu \mathrm{L})$ and $10 \mu \mathrm{L}$ methanolic solutions of extracts were spotted by Desaga TLC AS30 applicator (Desaga, Heidelberg, Germany) $0.5 \mathrm{~cm}$ from each edge of the chromatographic plate and developed in two directions by use of DS-II developing chambers (Chromdes, Lublin, Poland). In the first direction, the plate was developed using non-aqueous solvent and after drying in air the same plate was developed using aqueous eluent in the direction perpendicular to the first one. In case of non-aqueous solutions, each plate was conditioned in eluent vapours during $15 \mathrm{~min}$ to avoid the demixing effect; in case of RP systems (aqueous eluents), plates were not conditioned. After drying, the plates were sprayed using Merck TLC sprayer using 2-(diphenylboryoxy)-ethylamine and PEG4000 (Merck, Darmstadt, Germany and photographed in Camag Cabinet UV lamp at 254 and 365 nm) of Fuji 8 mpx camera or sprayed by DPPH and photographed in day light.

Each separation was replicated three times.

\section{Results and Discussion}

On the basis of our recent experiments [10], the preliminary choice of optimal eluent systems for the separation of phenolic fraction was performed. Next, the new experiments were carried out to devise chromatographic systems for achieving full separation of standards used in the search. It was stated that optimal NP systems for separation of standards were: $40 \%$ propan-2-ol (iPrOH) in $n$ heptane $(n-\mathrm{Hp})(\mathrm{v} / \mathrm{v})$ and $50 \%$ tetrahydrofuran (THF) in $n$ heptane $(\mathrm{v} / \mathrm{v})$, whereas, optimal RP aqueous eluent was 50 and $40 \%$ methanol $(\mathrm{MeOH})$ in water, respectively. The $R_{F}$ vs. $R_{F}$ correlations were made to show the $2 \mathrm{D}$-TLC separation of standards using selected pairs of solvents (Fig. 1a, b). On the basis of these experiments, the two-dimensional separations of standards were performed as well as extracts of investigated plant materials. 
Fig. 4 Photographs of chromatographic cyanopropyl plates developed by 2D-TLC mode (I-50\% THF $+n$-Hp; II-40\% MeOH + water) with separated extract of Pulmonaria officinalis. $\mathbf{a}$ at $\lambda=366 \mathrm{~nm} ; \mathbf{b}$ at $\lambda=254 \mathrm{~nm} \mathbf{c}$ after derivatization with DPPH in daylight $\mathbf{d}$ after derivatization with Naturstoff reagent at $\lambda=$ $366 \mathrm{~nm}$. Numbers as in Table 1
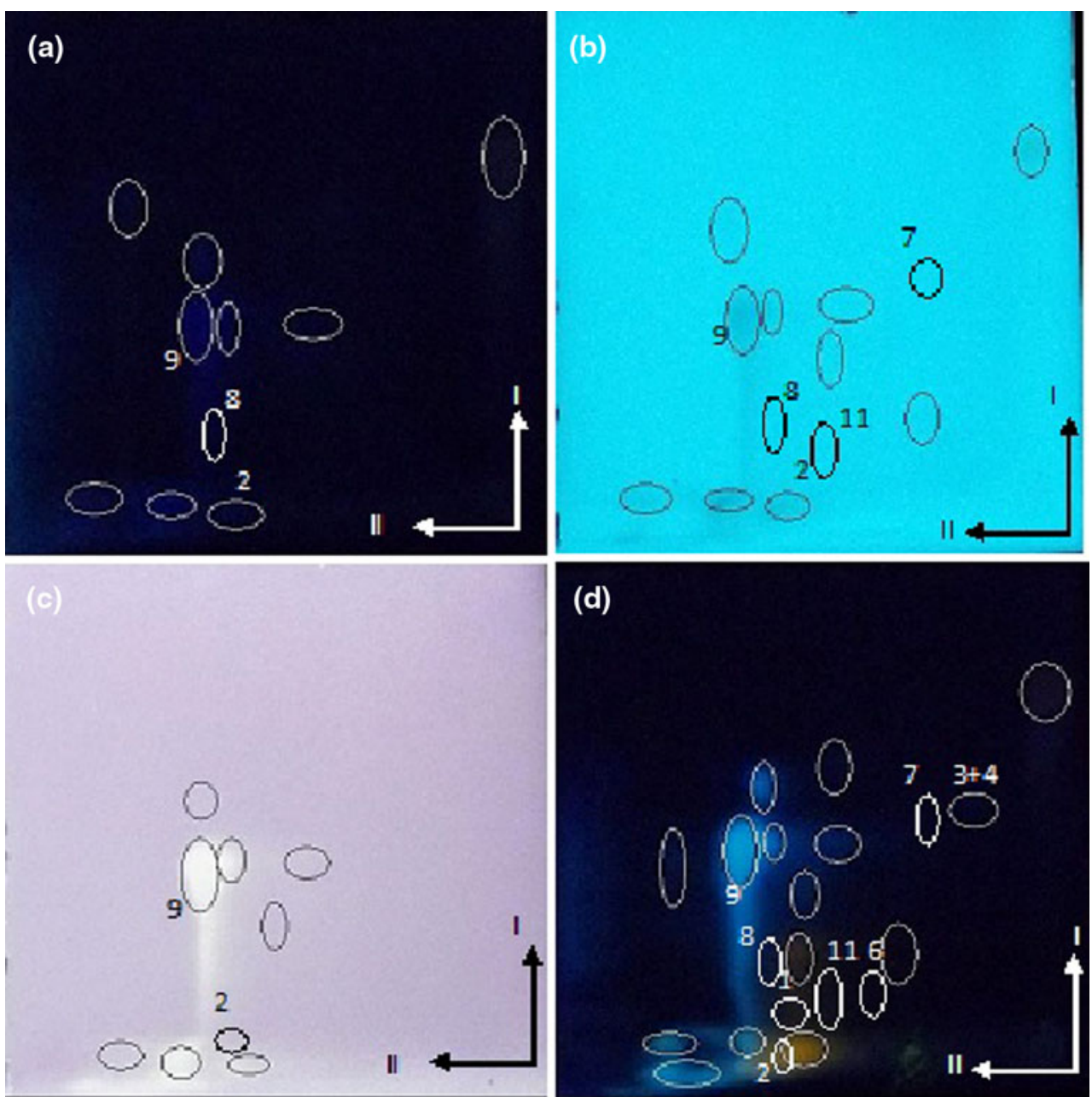

Documentation of chromatographic experiments includes pictures of plates photographed in Camag Cabinet UV lamp at 254 and $365 \mathrm{~nm}$ after derivatization with the Naturstoff reagent as well as in daylight after spraying with solution of DPPH by use of Fuji 8 mpx camera.

On the basis of chromatograms of standards (see Table 1), an attempt to investigate some extract components was performed. In the extract of $P$. hydropiper (Fig. 2) developed in system II, the following components were identified: naringin, hesperidin, naringenin, hesperetin, flavone, apigenin 7-glucoside, rutin, chlorogenic acid, myricetin and acacetin.

The extract of $B$. verrucosa contained naringin, hesperidin, hesperetin, apigenin 7-glucoside, rutin, chlorogenic acid, myricetin, hyperoside, acacetin and gallic acid (Fig. 3).

The extract from $P$. officinalis contained naringin, hesperidin, apigenin 7-glucoside, rutin, chlorogenic acid, myricetin, hyperoside, acacetin and gallic acid in system I and hesperidin, naringenin, hesperetin, apigenin 7-glucoside, rutin, chlorogenic acid and hyperoside in system II (see Fig. 4).
It should be also emphasized that all micro 2D-TLC chromatograms can be used as fingerprint for each investigated plant material because the differences in extracts' composition can be also noticed.

The antioxidative activity of fractions of phenolic compounds of investigated extracts can be performed using stable free radical DPPH (purple) giving with free radical scavengers yellow non-radical form. The effect of derivatizing using DPPH in examination of antioxidative activity of extracts is seen in Figs. 2, 3 and 4c. It is clearly seen that only some of the fractions' components exhibit such activity. There are especially chlorogenic acid (9), 7-Glucoside-Apigenin (6), rutin (8), hyperoside (12), myricetin (11), naringin (1), hesperidin (2), hesperetin (4), gallic acid (15), and quercetin (7).

\section{Conclusions}

Micro-2D-TLC is a useful technique for two-dimensional separation of herbal extracts. 
For separation of phenolic fraction of herb material in two-dimensional mode useful is system consisted of cyanopropyl layers developed with non-aqueous eluent composed of propan-2-ol or tetrahydrofuran and $n$-heptane in the first direction and aqueous one composed of methanol and water.

Micro-2D-TLC experiments give the information about composition as well as about antioxidative activity of plant extracts and are helpful in the construction of fingerprints of examined herbs.

Open Access This article is distributed under the terms of the Creative Commons Attribution License which permits any use, distribution, and reproduction in any medium, provided the original author(s) and the source are credited.

\section{References}

1. Poole CF, Poole SK (1989) Anal Chem 61:1257-1269
2. Poole CF, Fernando WPN (1992) J Planar Chromatogr 5:323-333

3. Zarzycki PK, Ślączka MM, Zarzycka MB, Bartoszuk MA, Włodarczyk E, Baran MJ (2011) J Steroid Biochem Mol Biol 127:418-427

4. Zarzycki PK, Ślączka MM, Zarzycka MB, Włodarczyk E, Baran MJ (2011) Anal Chim Acta 688:168-174

5. Zarzycki PK (2008) J Chromatogr A 1187:250-259

6. Zarzycki PK, Ohta H, Harasimiuk FB, Jinno K (2007) Anal Sci 23:1391-1396

7. Heinrich M, Barnes J, Gibbons S, Williamson EM (2004) Fundamentals of pharmacognosy and phytotherapy. Churchill Livingstone, Edinbrugh

8. Fraga CG (ed) (2010) Plant phenolics and human health biochemistry, nutrition, and pharmacology. John Wiley \& Sons Inc, Hoboken

9. Nishizawa M, Kohno M, Nishimura M, Kitagawa A, Niwano Y (2005) Chem Pharm Bull (Tokyo) 53:714-716

10. Hawrył MA, Robak M, Waksmundzka-Hajnos M (2011) J Chromatogr A 1218:2812-2819 